\title{
Effect of apoE genotype and dietary quercetin on blood lipids and TNF- $\alpha$ levels in apoE3 and apoE4 targeted gene replacement mice
}

\author{
Christine Boesch-Saadatmandi ${ }^{1}$, Siegfried Wolffram ${ }^{2}$, Anne Marie Minihane ${ }^{3}$ and Gerald Rimbach ${ }^{1 *}$ \\ ${ }^{1}$ Institute of Human Nutrition and Food Science, Christian Albrechts University Kiel, Hermann-Rodewald-Strasse 6, 24098 Kiel, \\ Germany \\ ${ }^{2}$ Institute of Animal Nutrition and Physiology, Christian Albrechts University Kiel, Hermann-Rodewald-Strasse 9, 24098 Kiel, \\ Germany \\ ${ }^{3}$ School of Chemistry, Food Biosciences and Pharmacy, University of Reading, Reading RG6 6AP, UK \\ (Received 29 May 2008 - Revised 2 September 2008 - Accepted 2 September 2008 - First published online 6 November 2008)
}

\begin{abstract}
The aim of this study was to determine the effect of dietary quercetin supplementation on blood lipids and TNF- $\alpha$ levels according to the apoE genotype in apoE3 and apoE4 targeted gene replacement mice. In a two-factorial design female apoE3 and apoE4 mice were fed semi-synthetic diets without (controls) and with quercetin $(2 \mathrm{mg} / \mathrm{g}$ diet $)$ for 6 weeks. Feeding the quercetin-supplemented diets significantly increased plasma levels of quercetin and isorhamnetin both in apoE3 and apoE4 mice. There was no significant effect of apoE genotype on plasma quercetin levels. ApoE3 and apoE4 transgenic mice exhibited similar plasma levels of apoE and cholesterol which were not significantly affected by dietary quercetin supplementation. In mice receiving the basal diet without quercetin supplementation, levels of TNF- $\alpha$ in whole blood stimulated ex vivo with lipopolysaccharide were higher in apoE3 as compared to apoE4 transgenic mice. Dietary quercetin significantly lowered levels of TNF- $\alpha$ by $44 \%$ in apoE3 mice relative to apoE3 mice receiving the unsupplemented diets. In apoE4 mice a moderate (20\%) but not significant decrease in TNF- $\alpha$ levels in response to the quercetin supplementation was evident. Following quercetin supplementation TNF- $\alpha$ levels were similar between apoE3 and apoE4 transgenic mice. Current findings indicate that apoE3 mice are more responsive to the TNF- $\alpha$ lowering properties of dietary quercetin supplementation as compared to apoE4 animals.
\end{abstract}

ApoE polymorphism: Quercetin: TNF- $\alpha$

Flavonoids are a large group of secondary plant metabolites with more than 6000 different compounds described to date. One important flavonoid subclass, the flavonols, and their major representative quercetin are ubiquitously distributed in plant foods and thus ingested in appreciable amounts with the daily diet. Particularly rich sources of quercetin are onions, apples and tea ${ }^{(1,2)}$. Epidemiological studies have revealed that the Mediterranean diet, which is particularly abundant in flavonoid-rich foods, correlates with increased longevity and a decreased incidence of $\mathrm{CHD}^{(3,4)}$. Dietary quercetin supplementation has recently been shown to lower platelet aggregation ${ }^{(5)}$ and blood pressure in man ${ }^{(6)}$. Furthermore, numerous cell culture studies indicate a potent antinflammatory activity of quercetin ${ }^{(7-11)}$.

ApoE is a polymorphic multifunctional protein with three common isoforms in man (E2, E3 and E4). ApoE3 is the wild-type and most common isoform, while apoE4 carriers account for about $25 \%$ of the Caucasian population ${ }^{(12)}$. Presence of the apoE4 allele is associated with a $40-50 \%$ higher risk of $\mathrm{CVD}^{(13)}$ and apoE4 is the major known genetic risk factor for maturity-onset Alzheimer's disease ${ }^{(14,15)}$.
Although apoE4 is strongly linked to both diseases, the molecular basis of these associations remains uncertain. Traditionally, the differences in disease risk have been attributed to the increased LDL-cholesterol (about $8 \%$ ) observed in apoE4 carriers, but it is becoming increasingly evident that this alone cannot explain the disease differential ${ }^{(16)}$. ApoE is not only synthesised by the liver, but also in the brain and by resident macrophages ${ }^{(17)}$ in the atherosclerotic wall, where it exerts atheroprotective actions, independent of its role in lipid metabolism ${ }^{(18)}$.

Our group has previously shown that the apoE genotype significantly affects the cellular inflammatory response in stably transfected murine macrophages. In apoE4 $v$. apoE3 macrophages higher levels of proinflammatory cytokines including TNF- $\alpha$ were evident following stimulation with lipopolysaccharide ${ }^{(19)}$. However, these findings refer to an in vitro situation and it is not known if the apoE genotype also affects biomarkers of inflammation in vivo. Furthermore, to the best of our knowledge no studies have examined the interaction between dietary flavonoids, apoE genotype and biomarkers of inflammation in vivo which is the aim of the present study. 


\section{Experimental methods}

Mice and diets

Female homozygous apoE3 and apoE4 targeted replacement mice (6-8 weeks old) 'humanized' for the apoE gene were purchased from Taconic Europe (Ry, Denmark). Generation of the apoE3 and apoE4 models was recently described ${ }^{(20)}$. Mice of both genotypes were assigned into one of two possible dietary groups ( $n 8)$, and were kept in macrolon cages at $21-25^{\circ} \mathrm{C}$, with a $12 \mathrm{~h}$ day-night cycle.

The diets and water were provided ad libitum for 6 weeks and live weight recorded weekly. Mice were kept according to the German Regulations of Animal Welfare with permission of the responsible authority. The semi-synthetic diet, based on maize starch $(15 \%)$, casein $(20 \%)$, sucrose $(33 \%)$ and butter fat $(21 \%)$ (so-called 'Western diet'), was purchased from Ssniff Special Diets (Soest, Germany). Diets were supplemented with 0 or $2 \mathrm{mg}$ quercetin/g diet, as dihydrate ( $\geq 98 \%$ purity; Roth, Karlsruhe, Germany).

\section{Sample collection and tissue preparation}

At the end of the dietary intervention period, mice were anaesthetised and decapitated. The blood was collected in EDTAtubes, and the plasma was separated by centrifugation $\left(8000 \mathrm{~g}, 4 \mathrm{~min}, 4^{\circ} \mathrm{C}\right)$ and stored at $-80^{\circ} \mathrm{C}$. An aliquot of EDTA-blood was diluted 1:10 with RPMI 1640 medium (supplemented with $10 \%$ fetal calf serum and $100 \mathrm{U} / \mathrm{ml}$ penicillin and $100 \mu \mathrm{g} / \mathrm{ml}$ streptomycin; all from PAA, Coelbe, Germany) and stimulated with $100 \mathrm{ng} / \mathrm{ml}$ lipopolysaccharide (from Salmonella enteritidis; Sigma, Deisenhofen, Germany) for $24 \mathrm{~h}$ as adapted from Miles et al. ${ }^{(21)}$. Accordingly, the supernatants were collected, centrifuged and cell-free samples were stored for further analysis at $-80^{\circ} \mathrm{C}$. Mice were genotyped according to the method of Zivelin et al. ${ }^{(22)}$.

\section{Cholesterol levels and apoE concentrations}

Plasma total cholesterol and apoE concentrations were determined on an ILAB 600 automatic analyser (Instrumentation Laboratories UK Ltd, Warrington, UK), using commercially available spectrophotometric kits (TC, Instrumentation Laboratories Ltd) and turbimetric immunoassay kits (Apolipoprotein E-HAWako; Alpha Laboratories Ltd, Eastleigh, UK).

\section{TNF- $\alpha$ levels}

TNF- $\alpha$ concentrations in the supernatants of whole-blood culture were determined by the mouse TNF $\alpha$ DuoSet ELISA development kit (R\&D Systems, Wiesbaden, Germany).

\section{Quercetin analysis}

Analysis of plasma quercetin and its methylated derivative isorhamnetin $\left(3^{\prime}-O\right.$-methyl-quercetin) was performed by HPLC with fluorescence detection after treatment with a $\beta$-glucuronidase-sulphatase mix as previously described ${ }^{(23)}$.

\section{Statistical analysis}

Statistical analysis was performed using SPSS version 13.0 (SPSS, Munich, Germany). Data were analysed for normality of distribution (Kolmogorow-Smirnov and Shapiro-Wilk tests) and equality of variance (Levene test) prior to two-way ANOVA, which was performed to test the independent effects of apoE genotype (apoE3 and apoE4), dietary quercetin levels ( 0 or $2 \mathrm{mg} / \mathrm{g} \mathrm{diet}$ ) and their interactions on the outcomes of interest. A $t$ test was used to compare means of quercetin-fed mice with their control genotype. Data are expressed as means with their standard errors and significance was accepted at $P<0.05$.

\section{Results}

Mice carrying the apoE3 and apoE4 genotype did not differ in live weight gain over the 6-week experimental period. Furthermore, there was no significant effect of dietary quercetin supplementation on final body weight in either the apoE3 or apoE4 transgenic mice (Table 1).

Feeding the quercetin-supplemented diets significantly increased plasma levels of quercetin and isorhamnetin both in apoE3 and apoE4 mice to a similar extent. In mice fed the control diet quercetin was detectable in low concentrations whereas its metabolite isorhamnetin could only be determined in traces. There was no significant effect of apoE genotype on plasma quercetin and isorhamnetin levels (Table 1).

Table 1 shows mean values of plasma apoE and total cholesterol according to apoE genotype and dietary quercetin supplementation. ApoE3 and apoE4 transgenic mice exhibited similar plasma levels of both apoE and cholesterol which were not significantly affected by dietary quercetin. In mice receiving the basal diet without quercetin supplementation, levels of $\mathrm{TNF}-\alpha$ in whole-blood supernatants after lipopolysaccharide stimulation were higher in apoE3 as compared to apoE4 transgenic mice. Dietary quercetin significantly lowered levels of TNF- $\alpha$ by $44 \%$ in apoE3 mice $(P=0 \cdot 0015)$. In apoE4 mice only a moderate decrease in TNF- $\alpha(20 \%)$ due to dietary quercetin was evident. Following quercetin supplementation TNF- $\alpha$ levels were similar between apoE3 and apoE4 transgenic mice.

\section{Discussion}

Most studies in the literature reporting anti-inflammatory activities of quercetin were conducted in vitro using quercetin concentrations in the cell culture medium of $1-50 \mu \mathrm{mol} / \mathrm{l}^{(8,9)}$. In the present study plasma levels of quercetin of $3 \cdot 0-3 \cdot 3 \mu \mathrm{mol} / \mathrm{l}$ were measured. Interestingly, this concentration was sufficient to induce a significant decrease in TNF- $\alpha$ levels after ex vivo stimulation of whole blood with lipopolysaccharide in apoE3 transgenic mice. The dietary quercetin level used in the present mouse study was set to be $2 \mathrm{mg} / \mathrm{g}$ diet, which refers to a quercetin concentration of approximately $400 \mathrm{mg} / \mathrm{kg}$ body weight. This is many times higher than quercetin concentrations which can be normally achieved due to a flavonoid-rich diet in $\operatorname{man}^{(24)}$. However, it needs to be taken into account that quercetin is also available in a purified form and commercially available quercetin supplements often contain quercetin concentration in a range between 500 and $1000 \mathrm{mg} /$ capsule $^{(25)}$.

In the present study a targeted gene replacement model was used to study interactions between apoE genotype and dietary 
Table 1. Effect of apoE genotype and dietary quercetin on final body weight, plasma levels of quercetin, blood lipids and TNF- $\alpha$ levels in apoE3 and apoE4 targeted gene replacement miceł

(Mean values with their standard errors)

\begin{tabular}{|c|c|c|c|c|c|c|c|c|}
\hline & \multicolumn{4}{|c|}{ ApoE3 genotype } & \multicolumn{4}{|c|}{ ApoE4 genotype } \\
\hline & \multicolumn{2}{|c|}{ Control diet } & \multicolumn{2}{|c|}{ Quercetin diet } & \multicolumn{2}{|c|}{ Control diet } & \multicolumn{2}{|c|}{ Quercetin diet } \\
\hline & Mean & SEM & Mean & SEM & Mean & SEM & Mean & SEM \\
\hline Initial body weight (g) & $18 \cdot 6$ & 0.54 & $18 \cdot 2$ & 0.54 & $17 \cdot 8$ & 0.40 & $17 \cdot 8$ & 0.34 \\
\hline Final body weight $(\mathrm{g})$ & $26 \cdot 5$ & 2.00 & 23.6 & 0.65 & $24 \cdot 3$ & 1.45 & 23.6 & 0.65 \\
\hline Quercetin ( $\mu \mathrm{mol} / / \mathrm{l}$ plasma) $(n 5)$ & 0.421 & 0.08 & $3 \cdot 32^{\star \star \star}$ & 0.38 & 0.333 & 0.02 & $2.99+\dagger$ & 0.49 \\
\hline Isorhamnetin ( $\mu \mathrm{mol} / / \mathrm{l}$ plasma) $(n 5)$ & 0.001 & 0.00 & $4 \cdot 86^{\star \star}$ & 0.65 & 0.048 & 0.01 & $4 \cdot 69+\dagger$ & 1.00 \\
\hline Cholesterol (mmol// plasma) ( $n$ 5) & 2.96 & 0.28 & 3.48 & 0.10 & 3.24 & 0.29 & 3.41 & 0.29 \\
\hline ApoE $(\mu \mathrm{g} / \mathrm{ml}$ plasma) $(n 5)$ & $36 \cdot 3$ & 3.05 & 37.6 & 2.36 & $34 \cdot 2$ & 1.82 & $39 \cdot 4$ & 3.57 \\
\hline TNF- $\alpha$ (pg/ml blood) $(n 8)$ & 44.5 & 3.06 & $26 \cdot 2^{\star \star}$ & $3 \cdot 13$ & $32 \cdot 8$ & 5.98 & $26 \cdot 5$ & 1.68 \\
\hline
\end{tabular}

Mean values were significantly different from those of the apoE3 control group $\left(t\right.$ test): ${ }^{* \star} P<0.01,{ }^{* \star \star} P<0.001$.

Mean values were significantly different from those of the apoE4 control group $(t$ test): $\uparrow \dagger P<0.01$.

ҒFor details of procedures, see Experimental methods. ApoE3 and ApoE4 targeted gene replacement mice were fed a diet without (control; $0 \mathrm{mg} / \mathrm{g}$ diet) or supplemented (quercetin diet; $2 \mathrm{mg} / \mathrm{g}$ diet) with quercetin for 6 weeks. Using two-way ANOVA, significant quercetin effects were observed for plasma quercetin $(P<0.001)$, isorhamnetin $(P<0.001)$ and TNF- $\alpha(P<0.01)$.

quercetin. It is well documented that human apoE4 carriers exhibit lower levels of the apoE isoprotein as compared to man carrying the E3 allele ${ }^{(26)}$. Thus in man it is generally difficult to differentiate between genotype effects and effects which may be related to differences in the circulatory levels of the apoE isoprotein. Since our targeted gene replacement mice exhibited almost identical amounts of plasma levels of apoE3 and E4, respectively, potential differences in blood lipids and biomarkers of inflammation in the present mouse study are therefore related to the apoE3 and $\mathrm{E} 4$ isoprotein per se.

Human studies investigating the impact of apoE genotype on plasma lipid levels have reported mixed findings, with 5-10\% higher total- and LDL-cholesterol levels typically evident in E4 carriers $^{(27,28)}$, an effect which has been attributed to a number of mechanisms including increased dietary cholesterol absorption, decreased hepatic LDL receptor-mediated uptake and an increase in the production rate of LDL from its precursor VLDL. ApoE3 and apoE4 transgenic mice exhibited similar plasma levels of apoE and cholesterol which were not significantly affected by dietary quercetin supplementation. Cholesterol homeostasis and LDL receptor activity may also be affected by quercetin $^{(29,30)}$. However, in vivo effects of quercetin supplementation on human plasma lipid profile are inconsistent as well. No changes in plasma lipids were observed after quercetin supplementation in healthy subjects ${ }^{(31,32)}$ whereas markedly lower blood lipids were found when quercetin (as grape powder/grape juice) was given to pre- and postmenopausal women ${ }^{(33)}$ and to haemodialytic and healthy subjects ${ }^{(34)}$.

An important observation of the present study is that, although the differences did not reach statistical significance, apoE4 mice receiving the quercetin-free basal diet had $26 \%$ lower levels of $\mathrm{TNF}-\alpha$ as compared to apoE3 mice. However, the mouse data are in contradiction to our data in murine RAW264.7 macrophages where elevated levels of TNF- $\alpha$ were evident in E4 $v$. E3 macrophages ${ }^{(19)}$. Thus cell culture data regarding apoE genotype and inflammation may not be directly extrapolated to an in vivo situation. Furthermore, it needs to be taken into account that apoE produced by macrophages contributes only to $20 \%$ of the apoE produced in the human body whereas $80 \%$ of apoE is produced in the liver. In accordance with the present TNF- $\alpha$ data we found also higher levels of C-reactive protein ${ }^{(20)}$, another biomarker of inflammation, in apoE3 as compared to apoE4 transgenic mice.

Another finding of the present study is that dietary quercetin significantly decreased TNF- $\alpha$ levels in ex vivo-stimulated whole-blood samples of apoE3 but not apoE4 transgenic mice. Following quercetin supplementation in mice, TNF- $\alpha$ levels in apoE3 were lower than those in apoE4. Thus, based on the present findings, it seems likely that human apoE3 carriers may be more responsive towards a dietary intervention with flavonoids. Again, the present finding is in accordance with human data where supplementation with $\alpha$-linolenic acid had a stronger effect on biomarkers of inflammation in apoE3 $v$. apoE4 carriers ${ }^{(35)}$. Therefore, the apoE genotype may in part explain the large heterogeneity of studies regarding potential health effects of flavonoids in man where cohorts are not genotyped for apoE polymorphisms.

The underlying molecular and cellular mechanisms by which dietary quercetin has resulted in a decrease of secreted TNF- $\alpha$ may be related to its ability to inhibit the NF- $\kappa$ Bdependent signal transduction cascades as reported earlier ${ }^{(8)}$. Further studies are warranted to test the hypothesis whether $\mathrm{NF}-\kappa \mathrm{B}$ signalling in vivo is different in apoE3 $v$. apoE4 mice.

The present study reports, for the first time, that dietary quercetin differentially affects TNF- $\alpha$ levels in E3 and E4 targeted replacement mice. However, the study also has some limitations. First, the concentration of quercetin used in the present study was rather high; thus dose-dependent studies are warranted. Second, whole blood was stimulated with lipopolysaccharide to induce TNF- $\alpha$ secretion thus the inflammatory potential rather than the baseline level of circulatory TNF- $\alpha$ was determined. The model of ex vivo stimulation of whole blood has been previously used to evaluate the antiinflammatory potential of dietary phenolics ${ }^{(21)}$. Finally, the duration of the experiment was set to be 6 weeks. Therefore it would be interesting to test the hypothesis whether chronic feeding with lower doses of quercetin over a longer period of time would affect TNF- $\alpha$ and other inflammatory cytokines in relation to apoE genotype. Furthermore, human studies are warranted to study interactions between dietary flavonoids, apoE genotype and biomarkers of chronic inflammation. 


\section{Acknowledgements}

The project was supported by a grant of the German Ministry of Education and Science entitled 'Functional Foods for Vascular Health' (BMBF 0313856A). C. B.-S. and G. R. designed the study. C. B.-S., S. W. and A. M. M. performed the analyses. C. B.-S., A. M. M. and G. R. wrote, edited and reviewed the final manuscript. None of the authors have a known conflict of interest.

\section{References}

1. Hertog MG, Feskens EJ, Hollman PC, et al. (1993) Dietary antioxidant flavonoids and risk of coronary heart disease: the Zutphen Elderly Study. Lancet 342, 1007-1011.

2. Manach C, Scalbert A, Morand C, et al. (2004) Polyphenols: food sources and bioavailability. Am J Clin Nutr 79, 727-747.

3. Williamson G \& Manach C (2005) Bioavailability and bioefficacy of polyphenols in humans. II. Review of 93 intervention studies. Am J Clin Nutr 81, 243S-255S.

4. Erdman JW Jr, Balentine D, Arab L, et al. (2007) Flavonoids and heart health: proceedings of the ILSI North America Flavonoids Workshop, May 31-June 1, 2005, Washington, DC. J Nutr 137, 718S-737S.

5. Hubbard GP, Wolffram S, de Vos R, et al. (2006) Ingestion of onion soup high in quercetin inhibits platelet aggregation and essential components of the collagen-stimulated platelet activation pathway in man: a pilot study. Br J Nutr 96, 482-488.

6. Edwards RL, Lyon T, Litwin SE, et al. (2007) Quercetin reduces blood pressure in hypertensive subjects. J Nutr 137, 2405-2411.

7. Lotito SB \& Frei B (2006) Dietary flavonoids attenuate tumor necrosis factor alpha-induced adhesion molecule expression in human aortic endothelial cells. Structure-function relationships and activity after first pass metabolism. J Biol Chem 281, 37102-37110.

8. Nair MP, Mahajan S, Reynolds JL, et al. (2006) The flavonoid quercetin inhibits proinflammatory cytokine (tumor necrosis factor alpha) gene expression in normal peripheral blood mononuclear cells via modulation of the NF-kappa beta system. Clin Vaccine Immunol 13, 319-328.

9. Cho SY, Park SJ, Kwon MJ, et al. (2003) Quercetin suppresses proinflammatory cytokines production through MAP kinases and NF-kappaB pathway in lipopolysaccharide-stimulated macrophage. Mol Cell Biochem 243, 153-160.

10. Wadsworth TL \& Koop DR (1999) Effects of the wine polyphenolics quercetin and resveratrol on pro-inflammatory cytokine expression in RAW 264.7 macrophages. Biochem Pharmacol 57, 941-949.

11. Jung WJ \& Sung MK (2004) Effects of major dietary antioxidants on inflammatory markers of RAW 264.7 macrophages. Biofactors 21, 113-117.

12. Eichner JE, Dunn ST, Perveen G, et al. (2002) Apolipoprotein E polymorphism and cardiovascular disease: a HuGE review. Am J Epidemiol 155, 487-495.

13. Song Y, Stampfer MJ \& Liu S (2004) Meta-analysis: apolipoprotein E genotypes and risk for coronary heart disease. Ann Intern Med 141, 137-147.

14. Mahley RW, Weisgraber KH \& Huang Y (2006) Apolipoprotein E4: a causative factor and therapeutic target in neuropathology, including Alzheimer's disease. Proc Natl Acad Sci U S A 103, 5644-5651.

15. Cedazo-Minguez A (2007) Apolipoprotein E and Alzheimer's disease: molecular mechanisms and therapeutic opportunities. J Cell Mol Med 11, 1227-1238.

16. Davignon J, Gregg RE \& Sing CF (1988) Apolipoprotein E polymorphism and atherosclerosis. Arteriosclerosis 8, 1-21.
17. Wilson PW, Myers RH, Larson MG, et al. (1994) Apolipoprotein E alleles, dyslipidemia, and coronary heart disease. The Framingham Offspring Study. JAMA 272, 1666-1671.

18. Miyata M \& Smith JD (1996) Apolipoprotein E allele-specific antioxidant activity and effects on cytotoxicity by oxidative insults and beta-amyloid peptides. Nat Genet 14, 55-61.

19. Jofre-Monseny L, Loboda A, Wagner AE, et al. (2007) Effects of apoE genotype on macrophage inflammation and heme oxygenase-1 expression. Biochem Biophys Res Commun 357, 319-324.

20. Jofre-Monseny L, Huebbe P, Stange I, et al. (2008) Influence of apolipoprotein $\mathrm{E}$ genotype and dietary alpha-tocopherol on redox status and $\mathrm{C}$-reactive protein levels in apolipoprotein E3 and E4 targeted replacement mice. Br J Nutr 100, 44-53.

21. Miles EA, Zoubouli P \& Calder PC (2005) Differential anti-inflammatory effects of phenolic compounds from extra virgin olive oil identified in human whole blood cultures. Nutrition 21, 389-394.

22. Zivelin A, Rosenberg N, Peretz H, et al. (1997) Improved method for genotyping apolipoprotein E polymorphisms by a PCR-based assay simultaneously utilizing two distinct restriction enzymes. Clin Chem 43, 1657-1659.

23. Ader P, Wessmann A \& Wolffram S (2000) Bioavailability and metabolism of the flavonol quercetin in the pig. Free Radic Biol Med 28, 1056-1067.

24. Manach C, Williamson G \& Morand C (2005) Bioavailability and bioefficacy of polyphenols in humans. I. Review of 97 bioavailability studies. Am J Clin Nutr 81, 230S-242S.

25. Weldin J, Jack R, Dugaw K, et al. (2003) Quercetin, an overthe-counter supplement, causes neuroblastoma-like elevation of plasma homovanillic acid. Pediatr Dev Pathol 6, 547-551.

26. Mahley RW \& Rall SC Jr (2000) Apolipoprotein E: far more than a lipid transport protein. Апnи Rev Genomics Hum Genet 1, 507-537.

27. Minihane AM, Jofre-Monseny L, Olano-Martin E, et al. (2007) ApoE genotype, cardiovascular risk and responsiveness to dietary fat manipulation. Proc Nutr Soc 66, 183-197.

28. Minihane AM, Khan S, Leigh-Firbank EC, et al. (2000) ApoE polymorphism and fish oil supplementation in subjects with an atherogenic lipoprotein phenotype. Arterioscler Thromb Vasc Biol 20, 1990-1997.

29. Pal S, Ho N, Santos C, et al. (2003) Red wine polyphenolics increase LDL receptor expression and activity and suppress the secretion of ApoB100 from human HepG2 cells. J Nutr 133, 700-706.

30. Davalos A, Fernandez-Hernando C, Cerrato F, et al. (2006) Red grape juice polyphenols alter cholesterol homeostasis and increase LDL-receptor activity in human cells in vitro. $J$ Nutr 136, 1766-1773.

31. Chopra M, Fitzsimons PE, Strain JJ, et al. (2000) Nonalcoholic red wine extract and quercetin inhibit LDL oxidation without affecting plasma antioxidant vitamin and carotenoid concentrations. Clin Chem 46, 1162-1170.

32. Conquer JA, Maiani G, Azzini E, et al. (1998) Supplementation with quercetin markedly increases plasma quercetin concentration without effect on selected risk factors for heart disease in healthy subjects. J Nutr 128, 593-597.

33. Zern TL, Wood RJ, Greene C, et al. (2005) Grape polyphenols exert a cardioprotective effect in pre- and postmenopausal women by lowering plasma lipids and reducing oxidative stress. J Nutr 135, 1911-1917.

34. Castilla P, Echarri R, Davalos A, et al. (2006) Concentrated red grape juice exerts antioxidant, hypolipidemic, and antiinflammatory effects in both hemodialysis patients and healthy subjects. Am J Clin Nutr 84, 252-262.

35. Paschos GK, Yiannakouris N, Rallidis LS, et al. (2005) Apolipoprotein $\mathrm{E}$ genotype in dyslipidemic patients and response of blood lipids and inflammatory markers to alphalinolenic acid. Angiology 56, 49-60. 